\title{
Nonrandom association of genes and characters found in indica $\times$ japonica hybrids of rice
}

\author{
Yo-Ichiro Sato, \\ Ryuuji Ishikawa† and \\ Hiroko Morishima*
}

\author{
* National Institute of Genetics, Mishima, \\ Shizuoka-ken 411, Japan. \\ $\dagger$ Faculty of Agriculture, Hirosaki Univ., Hirosaki, \\ Aomori-ken 036, Japan.
}

Two subspecies of common rice, indica and japonica, are distinguishable by differences in alleles at a number of loci controlling biochemical and morphological traits. The nature of associations between twelve alleles and phenotypes was studied in both a sample of varieties and hybrid populations derived from a single "indica $\times$ japonica cross. Many of the associations found in varieties were not found in the $F_{2}$ population. The nine sets of associations observed in $F_{2}$ are explained by linkage. However, seven nonrandom associations were recovered in an $F_{5}$ population, derived in such a way as to minimize the effect of zygotic selection, which were not found in $F_{2}$. The genes involved in these associations were not genetically linked and young zygotes showed no differences in fitness between parental and recombined genotypes. These results suggest that increasing parental association of genes and characters in a hybrid population is most probably caused by gametic selection. This gametic selection could be a causal factor promoting the indicajaponica differentiation in rice varieties.

\section{INTRODUCTION}

Among cultivars of common rice (Oryza sativa L.), genes and characters tend to be associated in a certain manner, causing the indica-japonica differentiation. Oka (1958) defined the Indica and Japonica types as two varietal groups having associations of contrasting states in phenol reaction $(P h / p h)$, apiculus hair length, $\mathrm{KClO}_{3}$ susceptibility, and tolerance to cold and drought. Recently, Glaszmann (1987) reported that two major varietal groups represented by associations of alleles at 15 loci largely corresponded to the Indica and Japonica defined by Oka (1958).

The origin of the indica-japonica differentiation still remains as a puzzling question. The role of adaptation to different environmental conditions has been emphasized by many authors, while some internal factors have been suggested (Oka, 1958; Ikehashi and Araki, 1986).

In order to elucidate the factors causing nonrandom association of genes and characters which results in the indica-japonica differentiation among varieties, the pattern of their associations was observed in indica $\times$ japonica populations raised by a method designed to minimize distortion of genotypic frequencies by zygotic selection. Here we described patterns of association in twelve genes and characters in $F_{2}$ and $F_{5}$ populations, and discuss the factors causing such nonrandom associations.

\section{MATERIALS AND METHODS}

Hybrid populations used in this study were derived from a cross Ac. 419 (indica) $\times$ Ac. 504 (japonica). Ac. 419 was released by pure line selection in India. Ac. 504 is a Taiwanese cultivar Taichung 65 (T65) which was raised from a cross between two Japanese native cultivars. Ac. 419 and T65 are typical indica and japonica respectively, and have contrasting sets of alleles at a number of loci and phenotypes of characters (table 1). The $F_{2}$ population consisted of 200 individuals. The $F_{3}$ and $F_{4}$ populations were raised by the single-seed-descent (SSD) method in which seed for the next generation was prepared as bulked single seed from each plant of the previous generation. The $F_{3}$ and $F_{4}$ populations consisted of 188 and 172 plants, respectively. The $\mathrm{F}_{5}$ population was raised by bulking two seeds from each $\mathrm{F}_{4}$ individual, and about 300 plants were grown. 
Table 1 Gene and character associations causing indica-japonica differentiation in varietal population

\begin{tabular}{|c|c|c|c|}
\hline \multirow[b]{2}{*}{ Character } & \multirow[b]{2}{*}{ Gene $^{1}$} & \multicolumn{2}{|c|}{ Phenotype or allele } \\
\hline & & indica & japonica \\
\hline Phenol reaction & $(P h / p h)(I I)$ & Positive $(P h)$ & Negative $(p h)$ \\
\hline $\mathrm{KClO}_{3}$ susceptibility & Unknown & Susceptible & Resistant \\
\hline Apiculus hair length & $($ Aph/aph $)(\mathrm{I})$ & Short $(a p h)$ & Long $(A p h)$ \\
\hline Purple apiculus & $(C)(\mathrm{I})$ & Rare & Frequent \\
\hline Red pericarp & $(R c)(\mathrm{IV})$ & Frequent & Rare \\
\hline Black hull & $\begin{array}{l}(P h)(\mathrm{II}) \\
(B h-a, B h-b)(\mathrm{I})\end{array}$ & Sometimes & None \\
\hline Awnedness & Unknown & Rare & Sometimes \\
\hline \multicolumn{4}{|l|}{ Isozymes } \\
\hline Phosphoglucose isomerase- 1 & $(P g i-2)(I)$ & 1,2 & 1 \\
\hline Catalase-1 & $($ Cat-1) (I) & 1 & 2 \\
\hline Acid phosphatase-1 & $(A c p-1)(d-33)$ & -4 & +9 \\
\hline Esterase-2 & $($ Est-2) (I) & 1,2 & nul \\
\hline Aminopeptidase- 2 & $(A m p-2)(s u g)$ & 2 & 1 \\
\hline
\end{tabular}

${ }^{1}$ Linkage group is indicated in parenthesis.

All $F_{2}$ and $F_{5}$ plants were examined on a single plant basis for phenol reaction $(P h / p h$, linkage group II), susceptibility to potassium chlorate $\left(\mathrm{KClO}_{3}\right)$ at the two or three leaf stage (quantitative traits), apiculus hair length (in mm, $A p h / a p h$; see Sato, 1985), pericarp colour $(R c / r c$, linkage group IV), apiculus colour $(C / c$, linkage group I) and hull colour (black or straw, complementary action of $P h, B h-a$ and $B h-b)$, and presence or absence of awns (genes unknown). Methods for investigation were described by Sato et al. (1986). These plants were examined also for five enzymeencoding loci segregating in the hybrid populations. The five loci assayed were Est-2 (linkage group I), Pgi-2 (linkage group I), Amp-2 (linkage group sug), Cat-1 (linkage group I, but independent from Est-2 and Pgi-2) and Acp-1 (linkage group $d-33)$, by the method described by Ishikawa et al. (1987).

In addition, a sample of 200 native cultivars which were collected from various localities in Asia was also examined as a control population (varietal population). Records of the same characters and isozymes examined in hybrid populations were taken for each cultivar.

To check possible sources of selection operating in a breeding system, three characters representing adaptability i.e., (1) seed fertility, (2) germination rate and (3) seedling survival, were measured at the young zygote stage, using seeds from each $F_{5}$ plant. Seed fertility was studied as a measurement of zygotic mortality before seed maturity. Twenty seeds were planted in a nursery bed, and non-germinating seeds and surviving seedlings were counted to estimate selection due to seed dormancy and seedling survival, respectively.

\section{RESULTS}

\section{Character and gene associations in the varietal sample}

The pattern of association among 12 genes and quantitave characters, observed among varieties is illustrated in fig. 1. Solid and dotted lines indicate associations significant at 1 and 5 per cent levels, respectively, which are based on correlation

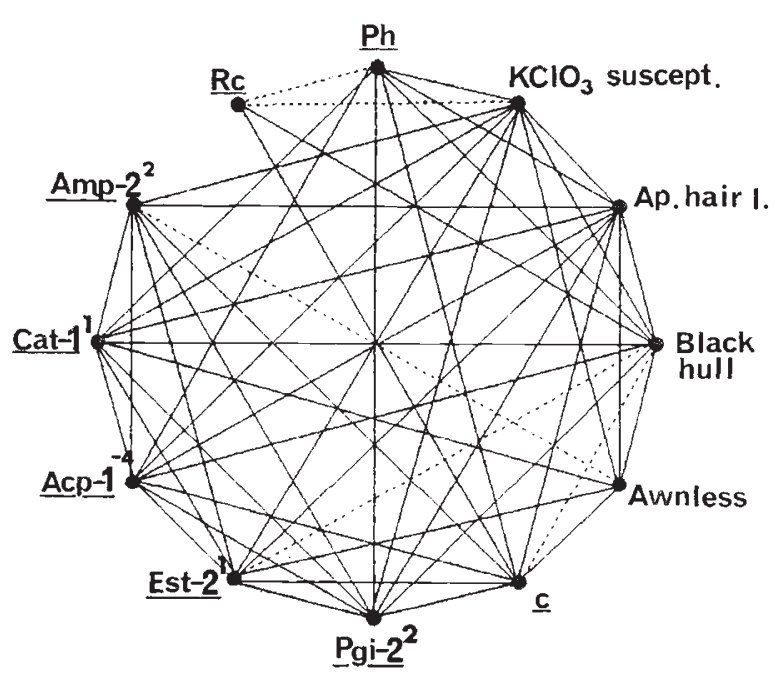

Figure 1 Pattern of association among genes and characters in varietal population. 
coefficients (between quantitative characters), chisquare values (between qualitative characters), or $t$ values (between qualitative and quantitative characters). Of the 66 possible combinations of genes and characters, 51 combinations (77 per cent) showed nonrandom association.

These associations give rise to two distinct groups of cultivars. The cultivars with the $P h$ allele tend to be susceptible to $\mathrm{KClO}_{3}$, tend to have short apiculus hairs (the aph allele) and have Cat-1 ${ }^{1}$, $A c p-1^{-4}$, and $A m p-2^{2}$ alleles. The cultivars with the ph allele, on the other hand, tend to have the opposite traits, i.e., resistance to $\mathrm{KClO}_{3}$, long apiculus hairs (the $A p h$ allele) and $C a t-1^{2}, A c p$ $1^{+9}$, and $A m p-2^{1}$ alleles. These two groups of cultivars also showed different allelic constitutions at the Pgi-2 and Est-2 loci (Oka, 1958; Morishima and Gadrinab, 1987). The former and latter combination of alleles are indica- and japonica-specific, respectively (table 1 ). These associations were confirmed by the present analysis.

\section{The pattern of association in} hybrid populations

The pattern of associations between the 12 genes and characters in the $F_{2}$ and $F_{5}$ populations are shown in fig. 2. Out of 66 possible combinations, nine in $F_{2}$ and 15 in $F_{5}$ showed nonrandom associations. The associations found in the $F_{2}$ are likely to be due to linkage (e.g., c, Est $-2^{1}$ and Pgi-2 ${ }^{1}$, cf. RGN 1, p. 46). Recently, the Aph locus was also found to belong to this linkage group (Sato et al., 1988 ). Black hull colour is controlled by three loci, $P h, B h-a$ and $B h-b$. Of these, $P h$ belongs to linkage group II. Either $B h-a$ or $B h-b$, or both are linked to Aph (Sato, unpublished data).
In the $F_{5}$ population, seven sets of associations which did not appear in $F_{2}$ were recovered. These included $P h$ and $a p h$, aph and $\mathrm{KClO}_{3}$ susceptibility, black hull and $R c$ (red pericarp), $P h$ and black hull, $P h$ and $R c, R c$ and $A m p-2^{2}$, and Est-2 ${ }^{1}$ and $A c p-1^{-4}$. In all cases except the $R c-A m p-2^{2}$ association, parental combinations of alleles found to be associated in indica and japonica (for instance, $P h a p h$ and $p h A p h$, respectively) were more frequent than non-parental combinations ( $P h A p h$ and phaph).

Differences in mortality at the gametic and young zygotic stages

Differences in the three characters between parental and recombinant types in $F_{5}$ are shown in table 2. Parental genotypes showed no advantage to recombinant types in the three characters. Seed fertility reflects differential success in the development of female gametes and fertilized embryos. Differences in seed germinability and seedling surviving rate result from selection at young zygote stage. This result indicates that the associations found in $F_{5}$ were not due to zygotic selection between the time of fertilization to seed germination. The nonrandom associations may well be caused by gametic selection, however.

\section{DISCUSSION}

The distinction between indica and japonica is based on multi-locus association as indicated by many authors (Oka, 1958, Sato et al., 1986; Glaszmann, 1987). They concluded that no single gene exists which is representative of indica-
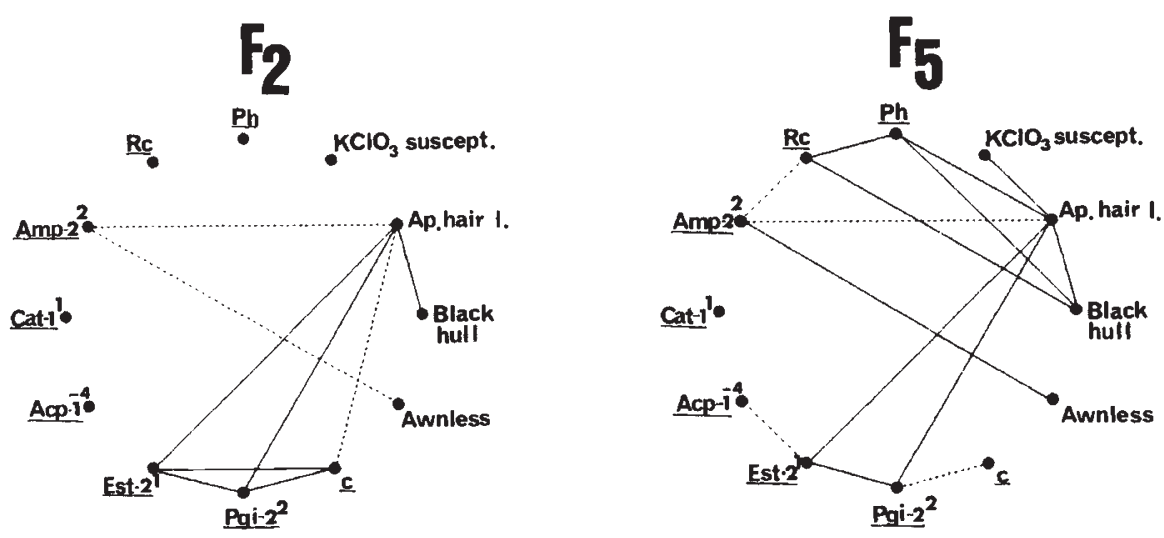

Figure 2 Associations among genes and characters in $F_{2}$ and $F_{5}$ populations. 
Table 2 Differences in seed fertility (SF), seed germinability (SG) and seedling survival (SS) between parental and recombinant genotypes defined by two loci and characters associated nonrandomly in $\mathrm{F}_{5}$

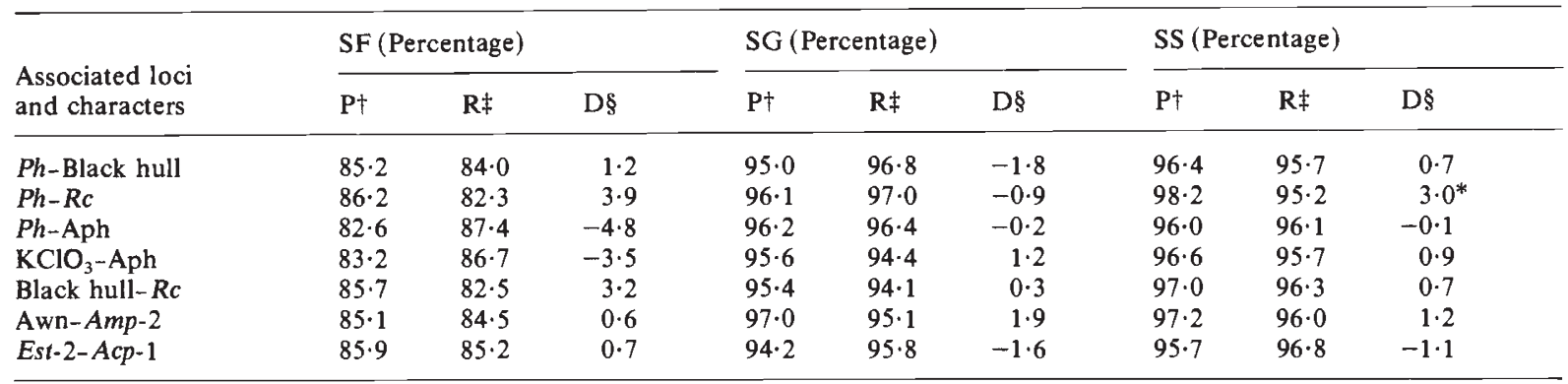

* Significant at 5 per cent level. No sign shows non-significant.

$\dagger$ Parental genotype

\$ Recombinant genotype

$\S$ Difference (parental - recombinant)

japonica differentiation. Therefore, mechanisms responsible for multi-locus association among varieties should be examined to elucidate factors causing the indica-japonica differentiation.

Nonrandom association between alleles at two or more loci is caused by various evolutionary forces such as gametic selection, zygotic selection, random drift, linkage and nonrandom mating in higher plants (Hedrick et al., 1978). Artificial selection also plays an important role in cultivated species.

In the indica-japonica hybrid populations, associations observed among the varietal sample largely disappeared in $F_{2}$. This indicates that the associations found among the varietal sample were partly caused by natural or artificial selections at the zygotic stage. Random drift may not be a causal factor because indica-japonica differentiation was also observed in African cultivers of $O$. sativa that have been established apart from Asian cultivers for a long time (de Kochko, 1987).

Associations found in the $F_{2}$ population are likely to be caused by linkage. Associations found in $F_{5}$ but absent in $F_{2}$, seven in total, could not, however, be explained by linkage. In fact, in five of the associations, the relevant loci are known to be carried on different chromosomes ( $P h$ and $R c$, $A c p-1$ and Est-2, Rc and $A m p-2, P h$ and $B h-a$, $B h-b$ and $R c$ and $B h-a, B h-b$ ). Other association sets $\left(\mathrm{KClO}_{3}\right.$ susceptibility-apiculus hair length and $P h$-apiculus hair length) appear also not to be explicable by linkage, although the linkage relationships of relevant genes are not fully known. In all cases except one, the nonrandom associations are brought about by an increase of parental genotypes relative to recombinants. No differences were observed in seed fertility, germination rate and seedling survival between parental and recombined genotypes (table 2), indicating that the nonrandom associations recovered in $F_{5}$ were not explained by selection due to differential fitness at the zygote stage.

In indica $\times$ japonica crosses, partial sterility of hybrids is frequently observed (e.g., Oka, 1953, 1974; Oka and Doida, 1962; Yokoo, 1984; Ikehashi and Araki, 1986) and results in gametic selection. Competition between pollen grains during pollen tube elongation which is caused by particular genes also results in gametic selection. Many have argued that gametic selection and nonrandom fertilization, e.g., competition among pollen grains and selective fertilization, play an important role in the evolution of higher plants (Ter Avanesian, 1978; Mulcahy, 1979; Hill and Load, 1986; Marshall and Ellstrand, 1986). It may be concluded that seven sets of associations found in $F_{5}$ were most probably due to gametic selection on the basis of the differential fertilizing abilities of gametes with different genotypes. It may be inferred that this trend for gametic selection acts as an internal mechanism for indica-japonica differentiation.

Acknowledgements The authors are grateful to Professors P. Hedrick, H. I. Oka, G. S. Khush and K. Yonezawa, for their encouragement and valuable advice.

\section{REFERENCES}

GLASZMANN, J. C. 1987. Isozymes and classification of Asian rice varieties. Theor. Appl. Genet., 74, 21-30.

HEDRICK, P. W., JAIN, S. K. AND HOLDEN, L. 1978. Multilocus systems in evolution. Evol. Biol., 11, 101-182. 
HILL, J. P. AND LOAD, E. M. 1986. Dynamics of pollen tube growth in the wild radish, Raphanus raphanistrum (Brassicaeae). I. Order of fertilization. Evolution, 40, 13281333.

IKEHASHI, H. AND ARAKI, H. 1986. Genetics of $F_{1}$ sterility in remote crosses of rice. In: Rice Genetics. (ed. by IRRI), 119-130.

ISHIKAWA, R., MORI, K., KINOSHITA, T. AND MORISHIMA, H. 1987. Expression pattern of isozyme genes in calli. Mem. Fac. Agr. Hokkaido Univ., 15, 371-379.

KOCHKO, A DE. 1987. Isozymic variability of traditional rice (Oryza sativa L.) in Africa. Theor. Appl. Genet., 73,675-682.

MARSHALL, D. L. AND ElLSTRAND, N. C. 1986. Sexual selection in Raphanus sativus: Experimental data on nonrandom fertilization, maternal choice, and consequencies of multiple paternity. Amer. Nat., 127, 446-461.

MORISHIMA, H. AND GADRINAB, L. U. 1987. Are the Asian common wild-rices differentiated into the indica and japonica types? In Hsieh, S. C. (ed.) Crop exploration and Utilization of Genetic Resources, Taiwan Prov. Taichung Distr. Agr. Improve. sta., Changhua, Taiwan, p. 11-20.

MULCAHY, D. L. 1979. The rise of angiosperms: A genecological factor. Science, 206, 20-23.

OKA, H. 1. 1953. The mechanism of sterility in the intervarietal hybrid. Jpn. J. Breed., 2, 217-224.
OKA, H. 1. 1958. Intervarietal variation and classification of cultivated rice. Ind. J. Genet. and Plt. Breed., 18, 79-89.

OKA, H. 1. 1974. Analysis of genes controlling $F_{1}$ sterility in rice by use of isogenic lines. Genetics, 77, 524-534.

OKA, H. I. AND DOIDA, Y. 1962. Phylogenetic differentiation of cultivated rice. XX. Analysis of the genetic basis of hybrid breakdown in rice. Jpn. J. Genet., 37, 24-35.

SATO, Y. 1. 1985. Genetic control of apiculus hair length. Rice Genet. Newslett. 2, 72-74.

SATO, Y. I., CHITRAKON, S., AND MORISHIMA, H. 1986. The Indica-Japonica differentiation of native cultivars in Thailand and neighbouring countries. In Napompeth, B. and Subhadrabandhu, S. (eds) New Frontiers in Breeding Researches, Kasetsart Univ., Bangkok, pp. 185-191.

SATO, Y. I., ISHIKAWA, R. AND MORISHIMA, H. 1987. Linkage analysis of $A p h$ gene responsible for apiculus hair length. Rice Genet. Newslett. 4, 74-75.

TER AVANESIAN, D. V. 1978. The effect of varying the number of pollen grains used in fertilization. Theor. Appl. Genet., $52,77-79$.

YOKOO, M. 1984. Female sterility in an Indica-Japonica cross of rice. Jpn. J. Breed., 34, 219-227. 